\title{
Evaluación de las propiedades psicométricas de la Escala de Satisfacción de las Necesidades Psicológicas Básicas en General con adultos españoles
}

\author{
Evaluation of the Psychometric Properties of the Scale of Satisfaction of Basic \\ Psychological needs in General with Spanish Adults
}

\author{
David González-Cutre \\ Ana C. Sierra \\ Carlos Montero-Carretero \\ Eduardo Cervelló \\ Jaime Esteve-Salar \\ Javier Alonso-Álvarez \\ Universidad Miguel Hernández de Elche, España
}

(Rec: 3 junio 2013 / Acept: 16 abril 2015)

\begin{abstract}
Resumen
Los postulados de la teoría de la autodeterminación defienden que para lograr una motivación más positiva y el bienestar es necesario satisfacer las necesidades psicológicas básicas: autonomía, competencia y relación. Para medir la satisfacción de estas necesidades en la vida, se ha creado la Escala de Satisfacción de las Necesidades Psicológicas Básicas en General (BNSG-S). Sus propiedades psicométricas han sido analizadas recientemente, aunque sólo con muestras de estudiantes universitarios. El objetivo del presente estudio fue analizar su validez y fiabilidad con una muestra de adultos españoles. En el estudio participaron 399 personas (202 hombres y 197 mujeres), con edades comprendidas entre 18 y 65 años. Los resultados del análisis factorial confirmatorio dieron apoyo a la validez de un modelo reducido de 16 ítems y efecto de método en los ítems negativos. La estructura factorial fue invariante por sexo y edad. Además, se obtuvieron valores adecuados de fiabilidad y estabilidad temporal. El análisis de las evidencias de validez externa mostró que la satisfacción de las tres necesidades psicológicas básicas se asociaba positivamente con la motivación autodeterminada hacia la actividad física y la satisfacción con la vida. Estos resultados dan apoyo a la utilización del instrumento en el contexto español.

Palabras clave: teoría de la autodeterminación, validación, satisfacción con la vida y actividad física.
\end{abstract}

\begin{abstract}
The postulates of self-determination theory advocate the satisfaction of basic psychological needs (autonomy, competence and relatedness) to achieve more positive motivation and well-being. The Basic Needs Satisfaction in General Scale (BNSG-S) was created to measure the satisfaction of these needs in life. Its psychometric properties have recently been analyzed, although only with samples of college students. The purpose of the present study was to analyze the validity and reliability of the BNSG-S with a sample of Spanish adults. Three hundred ninety-nine people (202 men and 197 women), aged between 18 and 65, participated in this study. The results of confirmatory factor analysis support the validity of a reduced model of 16 items and a negative-worded method effect. The factor structure was invariant across gender and age. It was also obtained acceptable values of reliability and temporal stability. The analysis of external validity evidences showed that the satisfaction of the three basic psychological needs was positively associated with self-determined motivation towards physical activity and satisfaction with life. These results support the utilization of the instrument in the Spanish context.

Keywords: self-determination theory, validation, satisfaction with life, physical activity.
\end{abstract}

\footnotetext{
Correspondencia: David González-Cutre, Centro de Investigación del Deporte, Universidad Miguel Hernández de Elche, Avenida de la Universidad s/n, 03202 Elche (Alicante), España. Tel: +34 9652221 62. E-mail: dgonzalez-cutre@umh.es

Nota del autor: La realización de este trabajo fue posible gracias al proyecto de investigación "Efectos físicos y psicológicos de un programa de ejercicio físico en pacientes intervenidos de cirugía bariátrica" (UEM2.11X), concedido en la convocatoria de ayudas para la realización de proyectos de investigación deportiva, Escuela de Estudios Universitarios Real Madrid-Universidad Europea de Madrid.
} 


\section{Introducción}

A lo largo de la historia, diferentes teorías han servido de marco de referencia a los investigadores preocupados por entender y explicar las necesidades psicológicas que tienen los seres humanos. Entre ellas, una de las más útiles y que más relevancia social ha alcanzado en la actualidad ha sido la teoría de la autodeterminación (TAD; Deci y Ryan, 2000; Ryan y Deci, 2000a). Esta teoría plantea la existencia de tres necesidades psicológicas innatas, universales y esenciales para la salud y el bienestar: autonomía, competencia y relación. La autonomía se refiere a la necesidad de sentir que tenemos capacidad de decidir acerca de nuestros propios actos, de expresar nuestras ideas y opiniones sin sufrir presiones externas, y de elegir libremente cómo vivir nuestra vida. La competencia se refiere a la necesidad de sentirse hábil y eficaz en las tareas desempeñadas, sentir éxito, demostrar nuestra capacidad y aprender nuevas habilidades. La relación alude a la necesidad de sentirse en buena sintonía con las personas cercanas, disfrutando de una vida social activa.

La TAD establece que la satisfacción de las necesidades es indispensable para el crecimiento psicológico de las personas (Vallerand y Verner-Filion, 2013), conduciendo hacia formas de motivación más autodeterminadas. De esta manera, los sentimientos de competencia, autonomía y relación llevarán a las personas a comprometerse en actividades por la diversión experimentada (motivación intrínseca), porque dichas actividades se integrarían en su estilo de vida (regulación integrada) o porque se atribuye cierta importancia a lo que se está realizando (regulación identificada). Sin embargo, la frustración de estas necesidades conduciría a implicarse en determinadas acciones únicamente por evitar sentimientos de culpabilidad (regulación introyectada) o por conseguir algún tipo de incentivo externo o recompensa, como el reconocimento de los demás (regulación externa), o bien llevaría directamente a no tener ninguna intención de desarrollar esa actividad al no verle la utilidad y el interés (desmotivación).

Las consecuencias más adaptativas se han relacionado con la satisfacción de las necesidades psicológicas básicas y con las motivaciones más autodeterminadas, por lo que desde hace años ha resultado de interés la evaluación de estas necesidades en contextos concretos, como el de la educación, la salud, el trabajo o la actividad física, e incluso a nivel global analizando la satisfacción de las necesidades psicológicas en la vida (Deci y Ryan, 2008). Concretamente, Gagné (2003) adaptó una escala de medida de la satisfacción de las necesidades en el trabajo (Kasser, Davey y Ryan, 1992), para medir las necesidades psicológicas básicas en la vida, desarrollando la Escala de Satisfacción de las Necesidades Psicológicas Básicas en General (Basic Needs Satisfaction in General Scale, BNSG-S).

Desde entonces, son muchos los trabajos que han utilizado este instrumento, a pesar de no haber testado previamente sus propiedades psicométricas (para una revisión, ver Johnston y Finney, 2010). Sólo recientemente, el trabajo realizado por Johnston y Finney (2010) comparó diferentes modelos para analizar la robustez psicométrica de este instrumento. Para ello realizaron un estudio con alumnos universitarios, en el que participaron 492 estudiantes de psicología, 2598 alumnos de primer curso en carreras universitarias y 1035 alumnos de segundo año. El objetivo del trabajo fue analizar la validez de constructo de la escala, para lo que se llevó a cabo una primera fase en la cual se realizó el análisis de la estructura factorial y una segunda fase en la que se analizó su validez externa.

En la primera fase se realizaron análisis factoriales confirmatorios (AFC) comparando un modelo unifactorial con otro que diferenciaba tres factores, en línea este último con los postulados de la TAD. El modelo unifactorial no obtuvo índices de ajuste aceptables tal y como se esperaba, lo que parecía apoyar que la BNSG-S es una escala multidimensional. En contra de lo esperado, el modelo basado en tres factores tampoco obtuvo índices aceptables. La mayoría de los desajustes estaban asociados a un efecto de método de los ítems redactados en negativo, a la redacción semántica de algunos de los ítems concernientes a autonomía, y a problemas de redundancia, covarianzas altas entre ítems y bajas varianzas explicadas, por lo que se modificó la escala original eliminando cinco ítems. Concretamente, los ítems 7 ("Me cierro bastante en mí mismo/a y no tengo muchas relaciones sociales") y 16 ("No hay mucha gente con la que tenga una relación muy cercana") se mostraron redundantes, por lo que se mantuvo sólo el 7 al considerarlo más representativo de la necesidad de relación. Además, se encontraron problemas teóricos y empíricos con diferentes ítems de autonomía (1, 4, 11, 14 y 20). Los ítems 1 ("Siento que soy libre para decidir por mí mismo/a cómo vivir mi vida") y 20 ("No tengo muchas oportunidades de decidir por mí mismo/a cómo hacer las cosas en mi vida diaria") parecen ser recíprocos, estando uno redactado en positivo y otro en negativo, por lo que se decidió eliminar el 20. El ítem 4 ("Me siento presionado/a en mi vida") parece estar escrito de forma muy general para considerar que únicamente se refiere a la autonomía (ya que la presión puede 
venir también determinada por la falta de competencia o relación); el ítem 11 mostró un rendimiento muy bajo y efecto de método; y el ítem 14 ("Las personas con las que me relaciono cada día tienen en cuenta mis sentimientos") parece más representativo de relación que de autonomía. Además, todos estos ítems reflejaron bajas varianzas explicadas, siendo por tanto eliminados. Por ello, los autores finalmente optaron por un modelo factorial de tres factores correlacionados y 16 ítems, con efecto de método asociado a los ítems negativos.

Para examinar la validez externa del instrumento, Johnston y Finney (2010) relacionaron las tres necesidades psicológicas básicas con diferentes dimensiones del bienestar, en la línea de definición del bienestar propuesta por Ryan y Deci (2000b), que considera a éste como un estado de funcionamiento óptimo de la persona, que consta de las dimensiones de autonomía, capacidad de crear entornos favorables, y capacidad de establecer relaciones positivas con los demás. Por otra parte, se analizaron también las relaciones entre las necesidades psicológicas y sentimientos de preocupación, como la anticipación de situaciones de fracaso y la ansiedad. Los resultados arrojaron relaciones más sólidas entre las necesidades y las dimensiones del bienestar, que entre éstas y los sentimientos de preocupación. Este patrón de correlaciones parece apoyar la diferenciación entre las dimensiones de la escala testada.

Posteriormente, Sheldon y Hilpert (2012) probaron diferentes modelos confirmatorios de la escala con estudiantes universitarios, demostrando de nuevo la necesidad de incluir un efecto de método en los ítems negativos de la BNSG-S para obtener índices de ajuste aceptables en el AFC. Además, analizaron la validez externa del instrumento, encontrando relaciones positivas entre la satisfacción de las necesidades psicológicas y el bienestar, contemplando la satisfacción con la vida como una de sus dimensiones. No obstante, estos autores identifican ciertas limitaciones en la BNSG-S, que les llevan a desarrollar un nuevo instrumento de medida de las necesidades psicológicas en la vida.

A la vista de estos resultados, y teniendo en cuenta que son escasos los trabajos que han analizado las características psicométricas de la BNSG-S y que los que lo han hecho sugieren seguir investigando sobre el particular (Johnston y Finney, 2010; Sheldon y Hilpert, 2012), el objetivo del estudio fue evaluar las características psicométricas de la BNSG-S en un nuevo contexto, el español, y con una nueva muestra, de adultos entre 18 y 65 años. Para ello se analizaron diferentes modelos confirmatorios, la invarianza por sexo y edad, fiabilidad, estabilidad temporal y evidencias de validez externa de la traducción al español de la escala. Para analizar las evidencias de validez externa se han analizado las relaciones entre la satisfacción de las necesidades en la vida y la motivación hacia la actividad física, teniendo en cuenta estudios previos (ThøgersenNtoumani y Ntoumanis, 2007) que han mostrado relaciones positivas entre la satisfacción de las necesidades psicológicas básicas en la vida y la motivación intrínseca en la actividad física, y negativas con las formas no autodeterminadas de motivación (regulación introyectada y externa). También, se han analizado las relaciones entre la satisfacción de las necesidades y la satisfacción con la vida, conocedores de las relaciones positivas mostradas entre ambas variables en estudios previos (Meyer, Enstrom, Harstveit, Bowles, y Beevers, 2007; Niemiec, Ryan y Deci, 2009; Sheldon y Hilpert, 2012).

\section{Método}

\section{Participantes}

En el estudio participaron 399 adultos (202 hombres y 197 mujeres) de dos provincias españolas, con edades comprendidas entre los 18 y 65 años $(M=31.30, D T=$ 11.31). Del total de participantes, 326 declararon practicar algún tipo de actividad física (caminar, correr, bailar, ir al gimnasio, practicar deporte), mientras que los 73 restantes declararon no practicar. Las frecuencias de práctica fueron diversas: 24 practicaban puntualmente, 159 lo hacián 2-3 días por semana y 143 practicaban más de 3 días semanales.

\section{Instrumentos}

Escala de Satisfacción de las Necesidades Psicológicas Básicas en General. Se utilizó la traducción al español de la Basic Needs Satisfaction in General Scale (BNSG-S, Gagné, 2003). La escala original se compone de un total de 21 ítems (ver Apéndice) que miden la satisfacción de las necesidades de competencia (seis ítems, e.g., "La mayoría de los días siento que tengo éxito en lo que hago"), autonomía (siete ítems, e.g., "Siento que soy libre para decidir por mí mismo/a cómo vivir mi vida") y relación (ocho ítems, e.g., "Realmente me gusta la gente con la que me relaciono"). En cada uno de los factores había tres ítems redactados de forma negativa. Los participantes debían contestar a todos los ítems pensando cómo se relacionaban con su vida e indicando cuán verdaderos eran para ellos en una escala tipo Likert del 1 (nada verdadero) al 7 (totalmente verdadero). 


\section{Cuestionario de Regulación de la Conducta en el} Ejercicio (BREQ-3): Para medir la motivación hacia la actividad física, se utilizó la versión española (González-Cutre, Sicilia y Fernández, 2010) del Behavioural Regulation in Exercise Questionnaire (Markland y Tobin, 2004; Wilson, Rodgers, Loitz y Scime, 2006). Este cuestionario está compuesto por 23 ítems que permiten evaluar las diferentes formas de motivación establecidas por la TAD: regulación intrínseca (e.g., "Porque disfruto con las sesiones de ejercicio"), integrada (e.g., "Porque considero que el ejercicio físico forma parte de mí"), identificada (e.g., "Porque para mí es importante hacer ejercicio regularmente"), introyectada (e.g., "Porque siento que he fallado cuando no he realizado un rato de ejercicio"), externa (e.g., "Porque mis amigos/ familia/pareja me dicen que debo hacerlo") y desmotivación (e.g., "No veo por qué tengo que molestarme en hacer ejercicio"). A los participantes se les preguntaba por qué practicaban o practicarían ejercicio y tenían que responder utilizando una escala Likert de 0 (nada verdadero) a 4 (totalmente verdadero). En el presente estudio se obtuvieron los siguientes valores de consistencia interna evaluados a través del alfa de Cronbach: .92 para regulación intrínseca, .93 para integrada, .83 para identificada, .69 para introyectada, .83 para externa y .85 para desmotivación. Además, se calculó el índice de autodeterminación (IAD), que nos indicaba cuán autodeterminada era la motivación hacia la actividad física de los participantes. Este índice se calcula multiplicando por 3 la regulación intrínseca, por 2 la integrada, por 1 la identificada, por -1 la introyectada, por - 2 la externa y por -3 la desmotivación, y sumando todos los productos. En este estudio, el IAD osciló entre - 18.33 y 21.75 .

Escala de Satisfacción con la Vida (SWLS). Se empleó la versión validada al español (Atienza, Pons, Balaguer, y García-Merita, 2000) de la Satisfaction With Life Scale de Diener, Emmons, Larsen y Griffin (1985). La escala se compone de cinco ítems (e.g., "En la mayoría de los aspectos, mi vida es como yo quiero que sea") que miden la satisfacción general con la vida de las personas, por medio de una escala Likert de 1 (muy en desacuerdo) a 5 (muy de acuerdo). En el presente estudio se obtuvo un valor alfa de Cronbach de .85 .

\section{Procedimiento}

La traducción de la BNSG-S se realizó utilizando técnicas de traducción inversa (Brislin, 1986). Un traductor bilingüe tradujo la escala del inglés al español y, posteriormente, dicha escala fue retro-traducida al inglés por un grupo de traductores independientes y bilingües. Este proceso se repitió hasta que la versión original y la versión inglesa retro-traducida fueron virtualmente idénticas. Se garantizó que el significado de los ítems se hubiera mantenido en el contexto español por medio de la evaluación de los mismos por parte de un grupo de investigación experto en psicología de la motivación y, en concreto, en la TAD. Además, la versión obtenida fue administrada de forma piloto a un pequeño grupo de adultos para verificar la comprensión de los ítems, sin presentarse ningún problema.

Una vez que la BNSG-S fue traducida, se administró junto con el BREQ-3 y la SWLS. El muestreo utilizado no fue aleatorio y consistió en solicitar la participación voluntaria en el estudio de diferentes personas captadas en la universidad, centros deportivos y centros sociales y de ocio. Se buscó un equilibrio en el tamaño muestral entre hombres y mujeres y entre personas de diferentes edades.

\section{Análisis de datos}

En línea con el trabajo que ha testado de forma más rigurosa las propiedades psicométricas de la BNSG-S (Johnston y Finney, 2010), se analizaron cuatro modelos factoriales a través del AFC; tres a partir de la versión original de 21 ítems: un modelo de un factor, un modelo de tres factores correlacionados, y un modelo con tres factores correlacionados y efecto de método asociado a los ítems negativos; y un último modelo reducido de 16 ítems con tres factores correlacionados y efecto de método asociado a los ítems negativos. Seguidamente se analizó la invarianza por sexo y por edad del último modelo, que fue el que mostró índices de ajuste aceptables, a través de un análisis multi-grupo. Para los diferentes análisis de ecuaciones estructurales, se utilizó el método de estimación de máxima verosimilitud con el procedimiento de bootstrapping. Este procedimiento puso de manifiesto la robustez de las estimaciones (Byrne, 2001), a pesar de la ausencia de normalidad de los modelos hipotetizados. Para analizar la bondad de ajuste del modelo se utilizaron los siguientes índices: coeficiente chi cuadrado $\left(\chi^{2}\right)$, ratio entre chi cuadrado y grados de libertad $\left(\chi^{2} / g l\right)$, CFI (Comparative Fit Index), IFI (Incremental Fit Index), RMSEA (Root Mean Square Error of Approximation) más su intervalo de confianza (IC) al 90\%, y SRMR (Standardized Root Mean Square Residual). Para el coeficiente $\chi^{2} / g l$ se suelen considerar como aceptables valores inferiores a 3 (Schermelleh-Engel, Moosbrugger y Müller, 2003). En cuanto al resto de índices, los puntos de corte establecidos como aceptables, atendiendo a las principales directrices que se siguen en el análisis de modelos de ecuaciones estructurales (Hu y Bentler, 1999), son los siguientes: valores de CFI e 
IFI iguales o superiores a .95, valores de RMSEA iguales o inferiores a $.06 \mathrm{y}$ valores de SRMR iguales o inferiores a .08 . No obstante, hay un consenso generalizado en considerar que estos valores son sólo orientativos (Marsh, Hau y Wen, 2004), y que para índices como el CFI y el IFI, criterios más antiguos (valores iguales o superiores a .90 ) son considerados como un límite inferior aceptable al coeficiente .81 .

A continuación se analizó la fiabilidad de la medida utilizando diferentes fórmulas (Johnston y Finney, 2010; McDonald, 1999). Para calcular la fiabilidad del factor autonomía, cuyos ítems eran unidimensionales, se utilizó la siguiente ecuación: $\left(\sum b_{i}\right)^{2} /\left(\sum b_{i}\right)^{2}+\sum e_{i}$ donde $b_{i}$ representa el peso de regresión sin estandarizar y $e_{i}$ la varianza del error sin estandarizar de los ítems. Para calcular la fiabilidad de los factores competencia y relación, que tenían un efecto de método asociado a los ítems negativos, la fórmula empleada fue: $\left(\sum b_{i}\right)^{2} /\left(\sum b_{i}\right)^{2}+\sum e_{i}+\left(\sum b_{j}\right)^{2}$, donde $b_{i}$ corresponde al peso de regresión sin estandarizar desde los ítems al factor, $e_{i}$ a la varianza del error sin estandarizar de los ítems, $y b_{j}$ al peso de regresión sin estandarizar desde el factor de efecto de método a los ítems negativos.

También se analizó la estabilidad temporal del instrumento, utilizando una pequeña muestra de personas dentro de la muestra total, a quienes se les administró la escala en dos ocasiones con una separación temporal de un mes. Para ello se calculó el coeficiente de correlación intraclase (CCI). Según Cicchetti (1994), valores del CCI inferiores a .40 se consideran pobres, entre .40 y .59 aceptables, entre .60 y .74 buenos, y por encima o iguales a .75 excelentes.

En último lugar, para analizar las evidencias de validez externa, se realizó un análisis de ecuaciones estructurales de las relaciones entre la satisfacción de las necesidades de autonomía, competencia y relación, la motivación autodeterminada hacia la actividad física y la satisfacción con la vida. Se proporcionaron los valores de las correlaciones obtenidas entre las diferentes variables, así como los estadísticos descriptivos. Todos los análisis de este estudio fueron efectuados con los paquetes estadísticos AMOS 18 y PASW Statistics 18, de SPSS Inc.

\section{Resultados}

\section{Análisis Factorial Confirmatorio (AFC)}

En primer lugar se testó un modelo de un factor compuesto por 21 ítems, encontrando que dicho modelo no se ajustaba a los datos, lo que refuerza la hipótesis de que la
BNSG-S es una medida multidimensional: $\chi^{2}(189, N=$ $399)=892.65, p<.001 ; \chi^{2} / g l=4.72 ; \mathrm{CFI}=.72 ; \mathrm{IFI}=.72$; RMSEA $=.097(90 \% \mathrm{CI}=.090-.103) ;$ SRMR $=.091 . \mathrm{En}$ segundo lugar se procedió a analizar un modelo de tres factores correlacionados, acorde con la estructura factorial de la escala original de 21 ítems. En este caso los índices de ajuste tampoco fueron aceptables: $\chi^{2}(186, N=399)=589.38$, $p<.001 ; \chi^{2} / g l=3.16 ; \mathrm{CFI}=.77 ; \mathrm{IFI}=.77 ; \mathrm{RMSEA}=.074$ $(90 \% \mathrm{CI}=.067-.081) ; \mathrm{SRMR}=.067$. Los pesos de regresión estandarizados fueron todos significativos, oscilando entre .34 y .65. Las correlaciones entre los tres factores fueron significativas: .85 entre competencia y autonomía, .77 entre autonomía y relación, y .76 entre competencia y relación. En tercer lugar se testó un modelo similar al anterior, pero introduciendo un efecto de método en los ítems negativos. Los índices de ajuste mejoraron, pero sin llegar a los puntos de corte establecidos para poder considerarlos aceptables: $\chi^{2}$ $(177, N=399)=423.41, p<.001 ; \chi^{2} / g l=2.39 ; \mathrm{CFI}=.86$; $\mathrm{IFI}=.86$; RMSEA $=.059(90 \% \mathrm{CI}=.052-.066)$; SRMR $=.054$. Los pesos de regresión estandarizados continuaron siendo significativos y las correlaciones entre los tres factores se atenuaron ligeramente: 81 entre competencia y autonomía, .75 entre autonomía y relación, y .70 entre competencia y relación.

En último lugar se testó el modelo propuesto por Johnston y Finney (2010), de tres factores correlacionados con efecto de método asociado a los ítems negativos y un total de 16 ítems (Figura 1), obteniendo unos índices de ajuste aceptables: $\chi^{2}(96, N=399)=181.96, p<.001 ; \chi^{2} /$ $g l=1.89 ; \mathrm{CFI}=.92 ; \mathrm{IFI}=.92 ; \mathrm{RMSEA}=.047(90 \% \mathrm{CI}=$ $.037-.058)$; SRMR $=.048$. Los pesos de regresión estandarizados fueron todos significativos, oscilando entre $.30 \mathrm{y}$ .73. Las correlaciones entre los tres factores también fueron significativas: .73 entre competencia y autonomía, .67 entre autonomía y relación, y .69 entre competencia y relación.

\section{Análisis de invarianza por sexo y edad}

Una vez encontrado el modelo factorial de la escala con mejor ajuste (tres factores correlacionados, 16 ítems y efecto de método en ítems negativos), se procedió a testar su invarianza factorial por sexo y por edad a través del análisis multi-grupo. Para ello se comparó un modelo sin invarianza (sin restricciones) con diferentes modelos a los que se les iba incorporando invarianza en sus diferentes parámetros de forma progresiva. No se encontraron diferencias significativas en $\chi^{2}$ entre el modelo sin restricciones y los modelos con invarianza en los pesos de medida y las covarianzas estructurales (Figura 1), dando apoyo así 


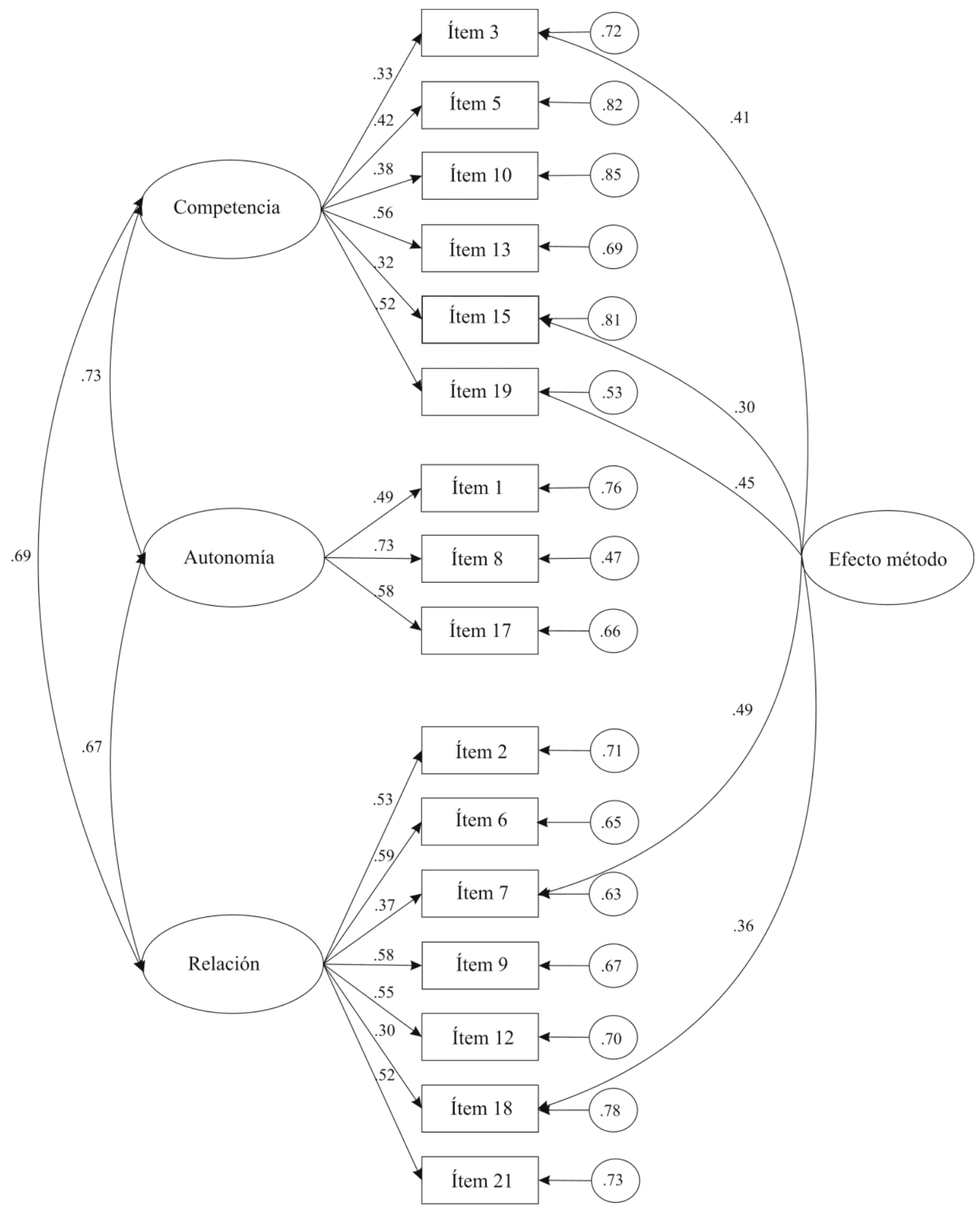

Figura 1. AFC de la BNSG-S de tres factores correlacionados con efecto de método asociado a los ítems negativos y un total de 16 items. Las elipses representan los factores y los rectángulos representan los diferentes items. Las varianzas residuales se muestran en los pequeños círculos. 
a la invarianza factorial por sexo. Además las diferencias en el CFI entre el modelo sin restricciones y los modelos con invarianza en los pesos de medida y las covarianzas estructurales fue inferior a .01, cumpliendo así también el criterio establecido por Cheung y Rensvold (2002) para considerar un modelo invariante.

Seguidamente, teniendo en cuenta el amplio rango de edad de la muestra (18-65 años) y tratando de comprobar si la estructura factorial de la escala era aplicable a diferentes grupos de edad, se analizó la invarianza factorial por edad. Se optó por dividir la muestra en tres grupos de edad, homogéneos en el número de participantes: 155 personas entre 18 y 24 años $(M=21.86, D T=1.69) ; 111$ personas entre 25 y 32 años $(M=28.04, D T=2.45)$; y 133 personas con más de 32 años $(M=45.03, D T=8.60)$. Esta decisión se tomó teniendo en cuenta que no había suficiente muestra entre 45 y 65 años para poder hacer las comparaciones de forma adecuada entre diferentes etapas vitales. En este caso no se encontraron diferencias significativas en $\chi^{2}$ entre el modelo sin restricciones y el modelo con invarianza en los pesos de medida, lo que supone un criterio mínimo (Byrne, Shavelson y Muthén, 1989; Milfont y Fischer, 2010) para aceptar la invarianza por edad. Además, las diferencias en CFI entre ambos modelos también fueron inferiores a .01 (ver Tabla 1).

\section{Análisis de fiabilidad y estabilidad temporal}

El análisis de fiabilidad contemplando en las ecuaciones los valores de los pesos de regresión y las varianzas del error sin estandarizar, tal y como se explica en el apartado de análisis de datos, reveló valores aceptables: .72 para competencia, .81 para autonomía y .82 para relación. Además, se llevó a cabo un análisis de estabilidad temporal con una submuestra de 94 personas (59 hombres y 35 mujeres), con edades comprendidas entre 18 y 63 años $(M=30.49$, $D T=12.51$ ), a las que se les administró la escala con una separación temporal de un mes. Se obtuvieron valores de CCI de .67 para competencia (Test: $M=3.98, D T=.69$; Retest: $M=4.05, D T=.68), .78$ para autonomía (Test: $M$ $=5.69, D T=.97$; Retest: $M=5.53, D T=1.02)$ y .49 para relación (Test: $M=4.87, D T=.47$; Retest: $M=4.85, D T=$ .49). Según Cicchetti (1994) estos valores se clasificarían como bueno, excelente y aceptable, respectivamente.

\section{Estadísticos descriptivos y evidencias de validez externa}

En la Tabla 2 se pueden ver los estadísticos descriptivos de las diferentes variables de estudio. Se obtuvieron valores entre moderados y moderadamente altos en la satisfacción de

Tabla 1. Análisis de invarianza multi-grupo.

\begin{tabular}{|c|c|c|c|c|c|c|c|c|c|}
\hline \multicolumn{10}{|c|}{ Invarianza por sexo } \\
\hline Modelos & $\chi^{2}$ & gl & $\chi^{2} / g l$ & $\Delta \chi^{2}$ & $\Delta g l$ & CFI & IFI & SRMR & RMSEA (IC 90\%) \\
\hline Modelo 1 & 334.97 & 192 & 1.74 & - & - & .88 & .88 & .067 & $.043(.035-.051)$ \\
\hline Modelo 2 & 360.71 & 209 & 1.72 & 25.74 & 17 & .87 & .87 & .071 & $.043(.035-.050)$ \\
\hline Modelo 3 & 369.38 & 216 & 1.71 & 34.40 & 24 & .87 & .87 & .075 & $.042(.035-.050)$ \\
\hline Modelo 4 & 404.07 & 232 & 1.74 & $69.09 *$ & 40 & .85 & .85 & .079 & $.043(.036-.050)$ \\
\hline \multicolumn{10}{|c|}{ Invarianza por edad } \\
\hline Modelos & $\chi^{2}$ & $\mathrm{gl}$ & $\chi^{2} / g l$ & $\Delta \chi^{2}$ & $\Delta g l$ & CFI & IFI & SRMR & RMSEA (IC 90\%) \\
\hline Modelo 1 & 441.12 & 288 & 1.53 & - & - & .87 & .88 & .069 & $.037(.030-.043)$ \\
\hline Modelo 2 & 481.36 & 322 & 1.49 & 40.23 & 34 & .86 & .87 & .078 & .035 (.029-.042) \\
\hline Modelo 3 & 510.04 & 336 & 1.51 & $69.91 *$ & 48 & .85 & .85 & .081 & $.036(.030-.042)$ \\
\hline Modelo 4 & 612.95 & 368 & 1.66 & $171.82 *$ & 80 & .79 & .79 & .083 & $.041(.035-.047)$ \\
\hline
\end{tabular}

Nota: Modelo 1 = sin restricciones; Modelo 2 = pesos de medida invariantes; Modelo 3 = covarianzas estructurales invariantes; Modelo 4 = residuos de medida invariantes. ${ }^{*} p<.05$ 
las necesidades psicológicas en la vida, atendiendo al rango de oscilación de la escala. Se encontró una mayor satisfacción de la necesidad de relación, seguida de la de autonomía y competencia. Además se obtuvieron puntuaciones más altas en las formas de motivación autodeterminadas (intrínseca, integrada e identificada) hacia la actividad física que en las no autodeterminadas (introyectada, externa y desmotivación). La puntuación media en el índice de autodeterminación fue entre moderada y moderadamente alta, considerando el rango de oscilación del índice en el presente estudio. La satisfacción con la vida obtuvo una puntuación superior al punto medio de la escala.

Para analizar las evidencias de validez externa se incluyeron en el modelo con efecto de método en los ítems negativos dos variables latentes, correlacionadas con los factores competencia, autonomía y relación. Dichas variables fueron la satisfacción con la vida y el índice de autodeterminación hacia la actividad física. No se incluyeron todas las formas de motivación en el modelo para mantener un equilibrio entre el número de parámetros y el tamaño muestral. La variable latente satisfacción con la vida estaba compuesta por cinco indicadores, representando cada uno la puntuación media de cada uno de sus ítems. La variable latente IAD estaba compuesta por dos indicadores que fueron calculados aplicando la fórmula del IAD a diferentes parcelas de ítems del BREQ-3. En cada factor se dividieron los ítems en dos grupos, cuyas medias fueron utilizadas para calcular un IAD 1 y un IAD 2, multiplicando por los diferentes pesos correspondientes (por 3 para cada parcela de regulación intrínseca, por 2 para las de integrada, por 1 para las de identificada, por -1 para las de introyectada, por -2 para las de externa y por -3 para las de desmotivación) y sumando todos los productos. El modelo obtuvo índices de ajuste aceptables: $\chi^{2}(215, N=399)=413.93, p<.001 ; \chi^{2} / g l=$ 1.92 ; CFI $=.93$; IFI $=.93$; $\mathrm{RMSEA}=.048(90 \% \mathrm{CI}=.041$ $.055)$; $\mathrm{SRMR}=.052$. Además, en línea de lo hipotetizado, la satisfacción de las necesidades psicológicas en la vida se asoció positiva y significativamente con la satisfacción en la vida y la motivación autodeterminada hacia la actividad física (ver Tabla 2).

Tabla 2. Estadísticos descriptivos y correlaciones entre las variables.

\begin{tabular}{|c|c|c|c|c|c|c|c|}
\hline Variables & M & DT & 1 & 2 & 3 & 4 & 5 \\
\hline 1. Competencia & 4.88 & .91 & - & $.75 * *$ & $.71 * *$ & $.70 * *$ & $.40 * *$ \\
\hline 2. Autonomía & 5.44 & 1.07 & & - & $.67 * *$ & $.55^{* *}$ & $.31 * *$ \\
\hline 3. Relación & 5.68 & .81 & & & - & $.41 * *$ & $.29 * *$ \\
\hline 4. Satisfacción con la vida & 3.76 & .81 & & & & - & $.21 * *$ \\
\hline 5. IAD & 9.75 & 8.81 & & & & & - \\
\hline 6. Intrínseca & 2.97 & 1.13 & & & & & \\
\hline 7. Integrada & 2.67 & 1.26 & & & & & \\
\hline 8. Identificada & 3.04 & 1.08 & & & & & \\
\hline 9. Introyectada & 1.89 & .79 & & & & & \\
\hline 10. Externa & 1.82 & .72 & & & & & \\
\hline 11. Desmotivación & .67 & .98 & & & & & \\
\hline
\end{tabular}

Nota: $* * p<.001$ 


\section{Discusión}

La BNSG-S ha sido utilizada de forma extendida en la investigación, aunque sólo recientemente se ha analizado su validez y fiabilidad de forma rigurosa (Johnston y Finney, 2010; Sheldon y Hilpert, 2012). El objetivo del presente estudio fue llevar a cabo una nueva evaluación de las propiedades psicométricas de este instrumento, en un nuevo contexto y con una nueva muestra. Este es el primer estudio que somete a análisis a la BNSG-S en España y que utiliza una muestra de adultos de 18 a 65 años. Estudios previos de validación utilizaron solamente muestras de estudiantes universitarios y sugerían realizar nuevas investigaciones en diferentes poblaciones, para examinar si las áreas de desajuste eran replicables, ya que determinadas modificaciones en la escala se podrían deber a las características idiosincráticas de la muestra (Johnston y Finney, 2010). Los resultados obtenidos en este estudio dan apoyo a la validez y fiabilidad de la versión reducida de 16 ítems de la BNSG-S propuesta por Johnston y Finney (2010).

En primer lugar se testaron diferentes modelos confirmatorios de la escala tratando de comprobar cuál de ellos se ajustaba mejor a los datos recogidos. El modelo unidimensional no obtuvo índices de ajuste aceptables, en línea con el trabajo de Johnston y Finney (2010), lo que refuerza la idea de que la BNSG-S es un instrumento multidimensional que mide tres constructos diferenciados: autonomía, competencia y relación. Este resultado es acorde a los postulados de la TAD, que establece la existencia de tres necesidades psicológicas básicas, y debe disuadir a los investigadores de utilizar un único índice que contemple las tres necesidades de forma conjunta como se hizo en investigaciones previas (e.g., Gagné, 2003; Niemiec et al., 2009).

A continuación se testaron dos modelos más, contemplando los 21 ítems de la versión original, uno de tres factores correlacionados y otro igual que éste pero introduciendo un efecto de método asociado a los ítems negativos. El modelo sin efecto de método no obtuvo índices de ajuste aceptables, tal como han mostrado investigaciones previas (Johnston y Finney, 2010; Sheldon y Hilpert, 2012). En lo que respecta al modelo de 21 ítems y efecto de método asociado a los ítems negativos, nuestros resultados revelan que el modelo tampoco se ajustaba a los datos. Johnston y Finney (2010) sí obtuvieron índices de ajuste aceptables a nivel global, pero hallaron otros problemas relacionados con grandes covarianzas residuales estandarizadas, que les llevaron a proponer un modelo reducido de 16 ítems.

Por tanto, el siguiente paso en este estudio fue testar la propuesta de Johnston y Finney (2010). Se eliminaron los cinco ítems que estos autores identificaron como problemáticos, teniendo en cuenta tanto aspectos teóricos, de redundancia y covarianzas altas entre ítems, como las bajas varianzas explicadas. Los resultados de este modelo en el presente estudio fueron satisfactorios, encontrándose índices de ajuste aceptables y correlaciones moderadas entre los tres factores. Por un lado, estos resultados dan apoyo a la presencia de un efecto de método asociado a los ítems negativos, lo que indica que éstos comparten una varianza común que no está explicada ni relacionada con sus respectivas variables latentes, sino que simplemente se asocia con el hecho de que los ítems están redactados negativamente. Esto podría sugerir que los ítems negativos (si su puntuación no se invierte) podrían estar midiendo la frustración de la necesidades, en línea de lo propuesto por recientes investigaciones que consideran que no es lo mismo la baja satisfacción de las necesidades que la frustración (Bartholomew, Ntoumanis, Ryan, Bosch y Thøgersen-Ntoumani, 2011; Sheldon y Hilpert, 2012). Por otro lado, los resultados dan apoyo a la necesidad de reducir la escala original de 21 a 16 ítems. No obstante, son necesarias nuevas investigaciones que analicen las diferentes estructuras factoriales y que traten de solventar los problemas encontrados con determinados ítems, depurándolos o proponiendo nuevos. En este sentido, se debe tener en cuenta que en la versión reducida de 16 ítems, el factor autonomía queda compuesto de sólo tres ítems y ninguno de ellos negativo, presentando un desajuste respecto a los otros dos factores.

En segundo lugar se analizó la invarianza factorial del modelo de 16 ítems y tres factores correlacionados con efecto de método. Los resultados sugieren que el modelo es invariante por sexo y edad, por lo que el instrumento se podría usar tanto en hombres como en mujeres y en diferentes grupos de edad, respetando su estructura factorial. Este es el primer estudio que, en nuestro conocimiento y hasta la fecha, ha analizado la invarianza factorial de la BNSG-S. Esta nueva prueba psicométrica del instrumento refuerza su validez, aunque no se debe olvidar que la invarianza obtenida fue sólo parcial, ya que no se daba en todos los parámetros del modelo. Además, para testar la invarianza factorial por edad se tuvieron que establecer tres grupos de edad homogéneos en tamaño muestral pero no en rango de edad. Esto conllevó que el grupo de mayor edad presentara un rango muy amplio (33-65 años), lo que nos lleva a sugerir que en futuros estudios se analice la invarianza por edad atendiendo a una división por etapas de la vida.

En tercer lugar se analizó la fiabilidad y estabilidad temporal de la escala. Teniendo en cuenta que el AFC presentaba un efecto de método, la fiabilidad no se calculó por medio 
del alfa de Cronbach, sino a través de dos ecuaciones que tenían en cuenta los pesos de regresión y las varianzas del error sin estandarizar. En este estudio se obtuvieron valores de fiabilidad aceptables (por encima de .70), a diferencia del estudio de Johnston y Finney (2010), en el que se obtuvieron valores bajos para competencia $(.55, .62$ y .60 para cada una de las tres muestras de estudio respectivamente) y autonomía $(.60, .68$ y .62), y valores aceptables para relación $(.78, .82$ y .80$)$, utilizando las mismas fórmulas. Respecto a la estabilidad temporal, en el presente estudio se encontró un valor de CCI bueno para competencia, excelente para autonomía, y aceptable para relación, con un intervalo de un mes entre las dos tomas. Estudios previos que evaluaron las propiedades psicométricas de la BNSG-S (Johnston y Finney, 2010; Sheldon y Hilpert, 2012) no testaron su estabilidad temporal, por lo que este estudio da un mayor apoyo a la fiabilidad de la escala.

En cuarto lugar se analizaron las evidencias de validez externa de la escala. Para ello se testaron las relaciones de la satisfacción de las necesidades psicológicas básicas en general con la satisfacción en la vida y la motivación autodeterminada hacia la actividad física. Los resultados revelaron correlaciones positivas y significativas, mostrando la percepción de competencia una correlación más fuerte con ambas variables, seguida de la autonomía y la relación. Estudios previos ya encontraron correlaciones positivas entre la satisfacción de las necesidades psicológicas y la satisfacción con la vida (Meyer et al., 2007; Niemiec et al., 2009; Sheldon y Hilpert, 2012). Estos resultados muestran que la satisfacción con la vida tiene más probabilidades de alcanzarse en aquellas personas que se sienten capaces de hacer cosas, que tienen nuevos aprendizajes, que sienten que tienen éxito, que son capaces de controlar su vida y tomar decisiones por sí mismas sin presiones externas, que pueden expresar libremente sus ideas y opiniones, y que cuentan con un círculo de personas que les apoyan, les valoran y les quieren.

Respecto a las relaciones entre la satisfacción de las necesidades psicológicas en la vida y la motivación hacia la actividad física, Thøgersen-Ntoumani y Ntoumanis (2007) encontraron resultados similares en una muestra de monitores de aeróbic. Las formas de motivación no autodeterminadas (regulación externa e introyectada) se correlacionaban negativamente con las necesidades psicológicas básicas, mientras que la motivación intrínseca se correlacionaba de forma positiva. Estos autores demostraron, además, que la insatisfacción de las necesidades podría relacionarse con una práctica de actividad física poco adaptativa y no autodeterminada, asociada a la búsqueda de la delgadez, la ansiedad físico-social y la insatisfacción corporal. Estos resultados podrían interpretarse desde dos puntos de vista. Por un lado, el hecho de no tener satisfechas las necesidades en la vida podría llevar a comprometerse con una práctica de actividad física a través de una motivación no autodeterminada que conllevara consecuencias negativas. Por otro lado, disfrutar de la práctica física podría llevar a tener más satisfechas las necesidades psicológicas en la vida, teniendo en cuenta los beneficios psicológicos que la actividad física ha demostrado (Biddle, Fox y Boutcher, 2000). En definitiva, el análisis muestra las evidencias de validez externa de la escala, ya que las relaciones obtenidas en este estudio entre la satisfacción de las necesidades psicológicas básicas en general, la satisfacción con la vida y la motivación autodeterminada hacia la actividad física, van en la línea de lo que encontraron estudios previos.

Resumiendo, los resultados de este estudio han dado apoyo a la validez y fiabilidad de la BNSG-S en un nuevo contexto (el español) y con una nueva muestra (adultos). El funcionamiento de la escala en poblaciones diferentes a la de estudiantes universitarios era desconocido hasta la fecha (Johnston y Finney, 2010). Sin embargo, las propiedades psicométricas encontradas han sido similares a las descritas por otros trabajos de validación (Johnston y Finney, 2010; Sheldon y Hilpert, 2012). El modelo que mejores propiedades presenta es el de 16 ítems y efecto de método. Los resultados muestran que las correlaciones entre los factores se mantienen estables a través de las poblaciones y los contextos, aunque los pesos de regresión continúan obteniendo valores bajos. Teniendo en cuenta la presencia de un efecto de método, que las varianzas explicadas de los ítems son pequeñas, que el factor autonomía está compuesto de pocos ítems, y que la invarianza factorial por sexo y edad es sólo parcial, se recomienda que futuros estudios continúen analizando las propiedades psicométricas de la escala, reformulando o creando nuevos ítems. Este estudio da un pequeño paso hacia la medida de calidad de las necesidades psicológicas básicas en la vida, aunque es necesario seguir trabajando para optimizar sus propiedades psicométricas. 


\section{Referencias}

Atienza, F. L., Pons, D., Balaguer, I. y García-Merita, M. (2000). Propiedades psicométricas de la Escala de Satisfacción con la Vida en adolescentes. Psicothema, 12, 314-319.

Bartholomew, K. J., Ntoumanis, N., Ryan, R. M., Bosch, J. A. y Thøgersen-Ntoumani, C. (2011). Self-determination theory and diminished functioning: The role of interpersonal control and psychological need thwarting. Personality and Social Psychology Bulletin, 37, 1459-1473.

Biddle, S. J. H., Fox, K. R. y Boutcher, S. H. (Eds.). (2000). Physical activity and psychological well-being. London: Routledge.

Brislin, R. W. (1986). The wording and translation of research instruments. En W. J. Lonner y J. W. Berry (Eds.), Field methods in educational research (pp. 137-164). Newbury Park, CA: Sage.

Byrne, B. M. (2001). Structural equation modeling with Amos: Basic concepts, applications, and programming. Mahwah, $\mathrm{NJ}$ : Erlbaum.

Byrne, B. M., Shavelson, R. J. y Muthén, B. (1989). Testing for the equivalence of factor covariance and means structures: The issue of partial measurement invariance. Psychological Bulletin, 105, 456-466.

Cheung, G. W. y Rensvold, R. B. (2002). Evaluating goodness-of-fit indexes for testing measurement invariance. Structural Equation Modeling, 9, 233-255.

Cicchetti, D. V. (1994). Guidelines, criteria, and rules of thumb for evaluating normed and standardized assessment instruments in psychology. Psychological Assessment, 6, 284-290.

Deci, E. L. y Ryan, R. M. (2000). The "what" and "why" of goal pursuits: Human needs and the self-determination of behaviour. Psychological Inquiry, 11, 227-268.

Deci. E. L. y Ryan, R. M. (2008). Self-determination theory: A macrotheory of human motivation, development, and health. Canadian Psychology, $3,182-185$.

Diener, E., Emmons, R., Larsen, R. J. y Griffin, S. (1985). The Satisfaction With Life Scale. Journal of Personality Assessment, 49, 71-75.

Gagné, M. (2003). The role of autonomy support and autonomy orientation in prosocial behavior engagement. Motivation and Emotion, 27, 199-223.

González-Cutre, D., Sicilia, A. y Fernández, A. (2010). Hacia una mayor comprensión de la motivación en el ejercicio físico: Medición de la regulación integrada en el contexto español. Psicothema, 22, 841-847.

Hu, L. y Bentler, P. M. (1999). Cutoff criteria for fit indexes in covariance structure analysis: Conventional criteria versus new alternatives. Structural Equation Modeling, 6, 1-55.

Johnston, M. M. y Finney, S. J. (2010). Measuring basic needs satisfaction: Evaluating previous research and conducting new psychometric evaluations of the Basic Needs Satisfaction in General Scale. Contemporary Educational Psychology, 35, 280-296.

Kasser, T., Davey, J. y Ryan, R. M. (1992). Motivation and employeesupervisor discrepancies in a psychiatric vocational rehabilitation setting. Rehabilitation Psychology, 37, 175-188.

Markland, D. y Tobin, V. (2004). A modification to Behavioural Regulation in Exercise Questionnaire to include an assessment of amotivation. Journal of Sport and Exercise Psychology, 26, 191-196.

Marsh, H. W., Hau, K-T. y Wen, Z. (2004). In search of golden rules: Comment on hypothesis-testing approaches to setting cutoff values for fit indexes and dangers in overgeneralizing Hu and Bentler's (1999) findings. Structural Equation Modeling, 11, 320-341.

McDonald, R. P. (1999). Test theory: A unified treatment. Mahwah, NJ: Erlbaum.

Meyer, B., Enstrom, M. K., Harstveit, M., Bowles, D. P. y Beevers, C. G. (2007). Happiness and despair on the catwalk: Need satisfaction, wellbeing, and personality adjustment among fashion models. The Journal of Positive Psychology, 2, 2-17.

Milfont, T. L. y Fischer, R. (2010). Testing measurement invariance across groups: Applications in cross-cultural research. International Journal of Psychological Research, 3, 111-121.
Niemiec, C. P., Ryan, R. M. y Deci, E. L. (2009). The path taken: Consequences of attaining intrinsic and extrinsic aspirations in post-college life. Journal of Research in Personality, 43, 291-306.

Ryan, R. M. y Deci, E. L. (2000a). Self-determination theory and the facilitation of intrinsic motivation, social development, and well-being. American Psychologist, 55, 68-78.

Ryan, R. M. y Deci, E. L. (2000b). The darker and brighter sides of human existence: Basic psychological needs as a unifying concept. Psychological Inquiry, 11, 319-338.

Schermelleh-Engel, K., Moosbrugger, H. y Müller, H. (2003). Evaluating the fit of structural equation models: tests of significance and descriptive goodness-of-fit measures. Methods of Psychological Research Online, 8, 23-74.

Sheldon, K. M. y Hilpert, J. C. (2012). The balanced measure of psychological needs (BMPN) scale: An alternative domain general measure of need satisfaction. Motivation and Emotion, 36, 439-451.

Thøgersen-Ntoumani, C. y Ntoumanis, N. (2007). A self-determination theory approach to the study of body image concerns, self-presentation and self-perceptions in a sample of aerobic instructors. Journal of Health Psychology, 12, 301-315.

Vallerand, R. J. y Verner-Filion, J. (2013). Making people's life most worth living: On the importance of passion for positive psychology. Terapia Psicológica, 31, 35-48.

Wilson, P. M., Rodgers, W. M., Loitz, C. C. y Scime, G. (2006). "It's who I am ... really!" The importance of integrated regulation in exercise contexts. Journal of Applied Biobehavioral Research, 11, 79-104. 


\section{Apéndice. Escala de Satisfacción de las Necesidades Psicológicas Básicas en General (BNSG-S)}

Por favor, lee cada uno de los siguientes ítems con atención, pensando cómo se relacionan con tu vida, e indica cómo de verdaderos son para ti.

1. Siento que soy libre para decidir por mí mismo/a cómo vivir mi vida. (AUT).

2. Realmente me gusta la gente con la que me relaciono (REL).

3. A menudo no me siento muy competente ( $\mathrm{COM}-$ ).

4. Me siento presionado/a en mi vida* (AUT -).

5. La gente que conozco me dice que soy bueno/a en lo que hago (COM).

6. Me llevo bien con la gente con la que me relaciono habitualmente (REL).

7. Me cierro bastante en mí mismo/a y no tengo muchas relaciones sociales (REL -).

8. Generalmente me siento libre para expresar mis ideas y opiniones (AUT).

9. Considero que la gente con la que me relaciono frecuentemente son mis amigos (REL).

10. Recientemente he sido capaz de aprender nuevas e interesantes habilidades (COM).

11. En mi vida diaria frecuentemente tengo que hacer lo que me dicen* (AUT -).

12. La gente que me rodea en mi vida se preocupa por mí (REL).

13. La mayoría de los días siento que tengo éxito en lo que hago (COM).

14. Las personas con las que me relaciono cada día tienen en cuenta mis sentimientos* (AUT).

15. En mi vida no tengo muchas oportunidades de demostrar lo capaz que soy (COM -).

16. No hay mucha gente con la que tenga una relación muy cercana* (REL -).

17. Siento que en mi vida cotidiana la mayoría de las veces puedo ser yo mismo/a (AUT).

18. Parece que no le gusto mucho a la gente con la que me relaciono frecuentemente (REL -).

19. A menudo no me siento muy capaz (COM -).

20. No tengo muchas oportunidades de decidir por mí mismo/a cómo hacer las cosas en mi vida diaria* (AUT -).

21. Generalmente la gente es bastante simpática conmigo (REL).

Nota. Los ítems que evalúan competencia han sido denotados con (COM), los que miden autonomía con (AUT), y los que miden relación con (REL). Los ítems negativos se destacan con un signo - después de dicha codificación. Los ítems señalados con * fueron eliminados en la versión de Johnston y Finney (2010) y en el presente estudio. 